\title{
OTHOTOMICS
}

Revista de economía, empresa y sociedad

Dosier "Género y empresa: hacia la igualdad real»

Coordinadora: Laura Lamolla Kristiansen

UNA MODALIDAD EN AUGE

\section{Mujeres y teletrabajo: más allá de la conciliación de la vida laboral y personal}

\author{
Ana Gálvez Mozo \\ Profesora agregada de los Estudios de Psicología y Ciencias de la Educación de la UOC
}

RESUMEN La problemática de la conciliación de la vida laboral, familiar y personal ha adquirido en las sociedades del siglo XXI una relevancia capital. Cada vez un mayor número de organizaciones se interesan en promover y promocionar este aspecto con el objetivo de mejorar el bienestar y la calidad de vida de sus trabajadores/as, así como para atraer y fidelizar talento, mejorar la productividad y, en definitiva, volverse más competitivos. En este contexto, una de las medidas más utilizadas para conseguir tales objetivos es el teletrabajo. Este artículo analiza, desde la percepción de las teletrabajadoras, si esta modalidad laboral constituye una estrategia eficiente de conciliación de la vida laboral, familiar y personal. La metodología utilizada ha sido cualitativa, en concreto se ha trabajado con entrevistas en profundidad y grupos de discusión. La muestra estaba compuesta por mujeres teletrabajadoras con algún tipo de carga familiar. Los principales resultados muestran que el teletrabajo para estas trabajadoras es una modalidad laboral que va mucho más allá de la conciliación de la vida laboral y familiar. Este es un tipo de lógica o dispositivo que resignifica las prácticas que despliegan y que conduce a la creación de nuevos significados de lo laboral y de la compatibilización con la vida doméstica, familiar y personal. Estas mujeres, a partir de la práctica del teletrabajo, elaboran un discurso crítico con el modelo laboral imperante, caracterizado por sus largas jornadas de trabajo que excluyen la posibilidad de una vida al margen de lo laboral. Utilizan las posibilidades de flexibilidad que les proporciona el teletrabajo para reivindicar su papel como profesionales y como madres, sin por esa razón renunciar a ninguno de ellos, y hacen uso del teletrabajo como herramienta para denunciar un mercado laboral que excluye todo lo relacionado con la esfera del cuidado.

PALABRAS CLAVE conciliación de la vida familiar, laboral y personal; organización flexible del trabajo; teletrabajo; género 


\title{
Women and teleworking: beyond work-life balance
}

\begin{abstract}
The difficulties in work-life balance has become greatly important in the societies of the 21st century. An evergrowing number of organisations are interested in promoting and encouraging this approach, with the objective of improving the well-being and quality of life of their male and female workers in addition to attracting and fostering talent, improving productivity and, ultimately, becoming more competitive. In this context, one of the most used measures for achieving such objectives is teleworking. This article analyses, from the point of view of female teleworkers, if this labour system constitutes an efficient strategy for the work-life balance. The methodology used has been qualitative, specifically working with indepth interviews and discussion groups. The sample was composed of women teleworkers with some form of family dependents. The principal results show that teleworking for these female workers is a labour system which goes far beyond work-life balance. This is a kind of logic or mechanism which redefines the unfolding practices and which leads to the creation of new meanings in the realm of work and in compatibilisation with domestic, family and personal life. By practising teleworking, these women are producing a critical discourse with the prevailing labour model, characterised by their long working days which exclude the possibility of a life at the margin of the world of work. They use the possibilities of flexibility which teleworking provides them in order to reclaim their roles as professionals and as mothers, and as a result do not give up either of these roles, making use of teleworking as a tool for revealing a labour market which excludes all that is related to the sphere of care.
\end{abstract}

KEYWORDS work-life balance; flexible organisation of work; teleworking; gender

\section{Introducción}

En los últimos años ha crecido el consenso entre instituciones internacionales, algunas tan relevantes como Naciones Unidas o la Organización Internacional del Trabajo, diferentes asociaciones de expertos y gobiernos locales, así como entre muchos países europeos, sobre la centralidad que la problemática de la conciliación de la vida laboral, familiar y personal ha adquirido en las sociedades del siglo XXI. Esto afecta, por supuesto, a los países industrializados, pero también a los países en vías de desarrollo. En el primer caso, se ha vuelto una cuestión clave vinculada con la masiva incorporación de las mujeres al mundo laboral, el aumento de las familias monoparentales y el envejecimiento progresivo de la población. En el segundo, constituye un problema de primera magnitud porque es el principal escollo que no permite articular el despliegue industrial acelerado que viven estos países con hábitos y tradiciones milenarias (Sorensen, 2017; Freeney y Stritch, 2017; Lewis y Beauregard, 2018).

Esta situación ha generado que muchas organizaciones se interesen formal e informalmente por la promoción de la conciliación no solo para mejorar el bienestar y la calidad de vida de sus trabajadores/as, sino también como medida para atraer y fidelizar talento, mejorar la productividad y, en definitiva, volverse más competitivos. En ese sentido se constata que las medidas utilizadas para cumplir tal finalidad son diversas, sin embargo, las más importantes se centran básicamente en la promoción de la flexibilidad. Así, World at Work (2015) señala que al menos el $80 \%$ de las organizaciones internacionales estarían ofreciendo en este momento algún tipo de organización flexible del trabajo. Estos nuevos modelos de organización (flexible work arrangements, en inglés -FWA-) se extienden rápidamente por todos los países de la Unión Europea y de la OCDE (Organización para la Cooperación 
y el Desarrollo Económico) y, como hemos mencionado, aspiran, ni más ni menos, a conjugar un aumento de la competitividad con una mejora de la vida de los/as trabajadores/as en todos sus ámbitos de expresión (Battisti y Vallanti, 2013; Eurofound, 2017).

En este contexto, el teletrabajo irrumpe como uno de los modelos que más expectativas ha generado para lograr la mencionada articulación. Este texto, basado en una investigación que ha durado más de un lustro y cuyos principales resultados pueden consultarse en Gálvez, Tirado y Alcaráz (2018, 2019), hace una revisión de las posibilidades que ofrece el teletrabajo y muestra la percepción que las mujeres teletrabajadoras tienen de esta modalidad laboral.

\section{Teletrabajo y conciliación}

España tiene una de las jornadas laborales más largas de la Unión Europea (Eurofound, 2017; Eurostat, 2016). Sin embargo, como es conocido, este hecho no repercute ni en una mayor productividad ni en un mayor número de horas trabajadas. La razón que explica tal paradoja es sencilla, se denomina «jornada partida». Esta consiste simplemente en dividir la actividad laboral con un descanso que usualmente es de 2 a 4 horas. Según el Instituto Nacional de Empleo, esta jornada es la más habitual en nuestro país y no presenta la tendencia de ir a una reducción significativa en los próximos años. Tal modalidad de organización de la actividad laboral diaria tiene importantes consecuencias sociales y familiares. Entre las más citadas destacan la ausencia de compatibilidad con los horarios escolares, lo que genera una gran complejidad y gasto económico en la logística familiar, y la dificultad para que los/as progenitores compartan tiempo y actividades con los/as hijos/as, especialmente cuando estos son pequeños, más allá de la franja del día que se restringe a las horas de la cena.

La «jornada partida» es un problema que se vincula en nuestro país directamente con una desigualdad de género muy elevada en la esfera doméstica. La mencionada jornada y una inflexibilidad extrema en los horarios laborales constituyen factores que facilitan que muchas mujeres opten por dedicarse plena o parcialmente al trabajo familiar y doméstico, abandonando sus carreras profesionales o incluso negándose a iniciarlas. Por todo esto, cada vez es más frecuente que organizaciones tanto del ámbito público como del privado busquen implementar medidas de flexibilidad laboral, entre las cuales el teletrabajo es una de las más populares. Esta suele presentarse asociada a una mejora de la conciliación y promete compaginar de forma muy ventajosa la dimensión espacio temporal de los/as trabajadores (Alina-Mihaela, Tuclea y Vrânceanu, 2019; Malenfant, 2009; Tietze y Musson, 2010; Lister y Harnish, 2010), y aparece, también, vinculada a una optimización de los recursos de la organización. Esto no quiere decir que la medida no tenga detractores. De hecho, en los últimos años algunos estudios han mostrado que el teletrabajo presenta graves problemáticas como pueden ser: a) la reificación de la distribución de roles de género; b) la sobrecarga de responsabilidad en las mujeres dado que la conciliación se convierte en un problema que se les atribuye directamente a ellas; c) dificultades en la promoción laboral (Kossek y Lautsch, 2017; Yao, Tan y llies, 2017; Hilbrecht, Shaw, Johnson y Andrey, 2013; Wheatley, 2012; Sorensen, 2011).

Nuestra investigación se sitúa en el centro de estos debates y en este artículo presentamos un análisis realizado con mujeres teletrabajadoras españolas en el que se examinó si la mencionada modalidad de trabajo ayudaba en la optimización de su jornada laboral y permitía desplegar estrategias eficientes de conciliación empleo-familia. Para ello se realizó un estudio cualitativo basado en la realización de entrevistas personales en profundidad y grupos de discusión. La muestra incluyó a 72 teletrabajadoras con responsabilidades familiares. El 20\% de las mismas teletrabajaban a tiempo completo y el restante $80 \%$ teletrabajaban a tiempo parcial. 


\section{2. ¿Combate el teletrabajo las largas jornadas laborales y permite la conciliación?}

Nuestro análisis presentó una dimensión que no esperábamos hallar en una investigación de este tipo y que no aparece especialmente mencionada en la literatura sobre el tema. Las entrevistadas señalaron claramente que una vez se habían organizado y habían aprendido lo que implica teletrabajar, esta modalidad laboral les permitía no solo reducir sus largas jornadas laborales, sino también optimizarlas y hacerlas más productivas. Sin embargo, cuando hablaban de tal cosa, su discurso no ponía el énfasis en cuestiones concretas relacionadas con las ventajas o las desventajas, sino que se centraba en dos elementos de lectura global y que implicaban una interpretación sociopolítica del fenómeno sobre el que eran interrogadas. El primero tiene que ver con la relación que ellas establecían entre lo laboral y lo domestico-familiar; y el segundo con el discurso absolutamente crítico y demoledor que planteaban contra el modelo laboral de sus organizaciones basado en jornadas laborales imposibles de compatibilizar con nada ajeno al trabajo y pensado especialmente para hombres o personas sin cargas familiares. Consideramos que estos dos hallazgos constituyen una parte muy interesante de nuestra investigación y a continuación los presentaremos con detalle.

\subsection{La relación entre lo laboral y lo doméstico-familiar}

Como hemos mencionado, la relación que las teletrabajadoras establecen entre lo laboral y lo doméstico-familiar constituye uno de los ejes centrales de sus discursos sobre el teletrabajo. Esta aparece como una relación basada en la apropiación y redefinición de los límites temporales y espaciales entre ambos ámbitos. Tal apropiación se concreta en tres modelos de definición: modelo de la simultaneidad, modelo de la intermitencia, y modelo de los compartimentos estancos. Estos no son excluyentes, es decir, nuestras entrevistadas los han utilizado en diferentes circunstancias, en función de las características y requerimientos de la situación.

\section{Modelo de la simultaneidad}

En el modelo de la simultaneidad, teletrabajo y familia se superponen en un mismo espacio y momento. Es una articulación efectiva si se dan una serie de circunstancias: a) tener hijos/as mayores; b) tener niños/as de corta edad, pero muy independientes; c) realizar tareas mecánicas; o d) la aparición de una simultaneidad impuesta por la situación. El siguiente verbatim ilustra muy bien este modelo:

«No, tengo un ordenador al lado, el portátil viejo, lo he puesto al lado en una mesita... y están allí, haciendo puzles y jugando. Y después también se cansan. Cogen el portátil y al comedor, donde tienen una especie de sala con juguetes, pues me pongo al lado los sábados por la mañana, ¿eh? Pues ellos... juegan ... y... y... lo que no podemos es estar en salas separadas, ¿eh? Porque ellos tienen... te tienen que ver». (GD3_006_colaboradora académica_teletrabajo parcial)

\section{Modelo de la intermitencia}

En la intermitencia, el teletrabajo y las tareas domésticas y familiares se suceden en intervalos de tiempo sucesivos, uno a continuación de otro, de forma intermitente y sin apenas mezclarse o superponerse. La mayoría de nuestras entrevistadas intercalaban en espacios y tiempos relativamente cortos el teletrabajo con las tareas domésticas. Manifestaron que precisamente esto constituye uno de los principales beneficios que aporta el teletrabajo como modalidad laboral. En este fragmento de una entrevista queda muy claro lo expuesto:

"Sí, cambia totalmente. Cuando estoy en casa, las tareas estas se combinan con poner lavadoras, preparar la comida, o preparar no sé qué. O sea: "Ahora hago una hora de no sé qué y después me pondré con...", o 
sea, vas combinando. Y cuando estoy en el despacho haciendo también tareas online estoy solo haciendo eso.» (EP_039_profesora universitaria_teletrabajo parcial)

\section{Modelo de compartimentos estancos}

En este modelo, el tiempo de teletrabajo y el dedicado a otras dimensiones de la vida de la trabajadora se separa de forma clara y rotunda. Los horarios tienen un principio y un fin muy bien definidos y ocupan un espacio muy delimitado del día. Las interferencias que puedan provocarse entre el trabajo, la familia y las tareas domésticas son mínimas o nulas y las entrevistadas manifestaron que tendían a mantener con todas sus energías esa compartimentación.

"Sí, sí. Se cierra la puerta y ya... ellos saben, si esa puerta está cerrada, que... a menos que haya un incendio y que me vengan a avisar, no pueden... molestar.» (EP_017_directora departamento_teletrabajo parcial)

Conviene añadir que, junto a la definición de estos modelos, también se expresaron factores o variables que facilitan el paso de uno de ellos a otro. Por ejemplo, las circunstancias familiares (enfermedades, cuidados imprevistos, accidentes domésticos, etc.) constituyen una variable que hacen que cambien de modelo para adaptarse a la circunstancia sobrevenida. También el tipo de objetivo o tarea que debe realizar la teletrabajadora puede hacer que se cambie de un modelo establecido durante un tiempo a otro que se adapta mejor a la nueva demanda. Por tanto, concluimos que los modelos descritos anteriormente son dependientes de la situación en la que se encuentra la teletrabajadora y absolutamente flexibles a la hora de ser modificados. En este sentido, resulta interesante señalar que las teletrabajadoras, en su uso de los modelos, muestran una apropiación del tiempo que no tiene nada que ver con la definición habitual que hacemos del mismo como algo discreto, lineal y objetivo. Nuestras entrevistadas lo resignificaban completamente al margen de variables técnicas u objetivas, lo hacían a partir de su experiencia y necesidades. Por lo tanto, lo convertían, a veces, en un tiempo circular que reverberaba sobre unas actividades domésticas que interferían la demanda de la organización, otras, como una línea de intensidad enorme que exigía renunciar a lo familiar para concentrarse en algo que debía solucionarse rápidamente, etc.

La aparición de estos modelos en los discursos de nuestras entrevistadas nos pone sobre la pista de un tema muy interesante. El teletrabajo es una modalidad de organización de las responsabilidades y objetivos laborales que implica mucho más que una mera reducción de la extensión de la jornada laboral. Los mencionados modelos, más allá de describir una rearticulación del límite entre trabajo y vida doméstica, señalan una resignificación total de la noción de «tiempo y espacio de trabajo». Al hacer tal cosa, también están cambiando su conceptualización de lo que supone un ambiente físico y simbólico para lo laboral. Por tanto, no resulta aventurado sostener que el teletrabajo es más una lógica o racionalidad para entender lo laboral que una simple técnica para reorganizar la vida de sus usuarias. El alcance de tal racionalidad aparece claramente expuesto en el siguiente apartado.

\subsection{El teletrabajo como resistencia al modelo laboral imperante}

Como hemos visto en el apartado anterior, los tres modelos de relación temporo-espacial que nuestras entrevistadas han descrito en su desempeño del teletrabajo están inextricablemente ligados a procesos de reflexión sobre sus jornadas laborales presenciales, sobre su práctica de teletrabajo y sus responsabilidades domésticofamiliares. Del mismo modo, están ligados también al ejercicio de toma de decisiones sobre cómo desarrollarlas y desde qué posición; y, por tanto, al inicio de cursos de acción sobre cómo estructurar y organizar su vida. Estos procesos definen a estas teletrabajadoras como agentes activos con capacidad para actuar e influir en el entorno laboral, familiar y social del que forman parte. Tal capacidad agentiva se asienta en los siguientes ejes: 
a) El teletrabajo y el conjunto de transformaciones espaciales y temporales que conlleva se convierten en una herramienta de emancipación, tal y como muestran los siguientes verbatims:

"La libertad de elegir cuando trabajar, de... y que los tiempos que aquí se veía más muerto, o menos, que te rendían más, allí en casa, lo aprovechas de otra... no hay tiempos muertos, no hay momentos que dices: ¿qué hago? No. Siempre hay algo que hacer, que sea para ti, que sea para el trabajo, que sea para los niños o para la casa. Entonces, de elegir cómo encajar bien tus momentos con los momentos de... de trabajo. La libertad de elegir cuándo trabajar, cómo trabajar...» (GD9_060_asistente dirección_teletrabajo tiempo completo)

El teletrabajo genera una autonomía altamente valorada y expresamente vinculada con la noción de libertad y la capacidad de autodelimitar las fronteras entre ámbitos de vida.

b) Teletrabajar se convierte en un sinónimo de crítica y rechazo del modelo de presencialidad imperante en las organizaciones de nuestro entorno. Nuestras entrevistadas muestran un completo rechazo de un modelo laboral con largas jornadas que obliga a no responsabilizarse de las obligaciones domésticas y familiares. El siguiente fragmento, como otros muchos que hemos hallado, señala cómo el modelo español detenta una estructura absurda que solo potencia una discriminación de género:

«Hombre, en cierto modo... siempre depende de la empresa en la que estés, pero, pero, suele limitar tu profes... tu promoción [...] cuando estás en una empresa, pues muchas veces lo que importa es que estés ahí. Que seas visible.» (EP_001_traductora_teletrabajo tiempo completo)

«Pero yo creo que al final en las empresas, y sobre todo en España, algo que... algo que no ocurre en otros países y que nos limita muchísimo, es la cantidad de horas que se trabaja. O sea, no tienes una jornada, o sea, porque si yo tuviera que tener un horario, mira, yo trabajo de nueve a seis, pues trabajo de nueve a seis, esto es imposible. O sea... es que en ninguna empresa tú puedes decir que trabajas de nueve a seis, yo no la conozco. En ninguna de las que he estado, es que... no. Tu disponibilidad para una empresa es todo el día. Es muy difícil eh... compaginar esto porque, si yo trabajo de nueve a seis, pues a lo mejor, no tengo ni que solicitar la reducción de jornada, porque digo bueno tengo un horario. Yo sé que a las seis me voy, tope cinco y media, seis me voy, como ocurre en cualquier sitio europeo, y aquí no... entonces eh... claro, al no tener... tú lo que quieres... es dejar pasta en la empresa... tu ni caso.» (EP_027_ingeniera_teletrabajo parcial)

c) El teletrabajo permite que no se renuncie ni a la faceta que abre la maternidad ni a una posible carrera profesional:

«Es cierto, pero a mí me encanta estar con los niños. Ahora hacemos un puzle, ahora hacemos no sé qué, me encanta... y me gusta mucho esto, y me gusta mucho mi trabajo. Porque al final dices: yo podría permitirme cinco años sin trabajar, económicamente. ¿Por qué lo haces? ¿Por el dinero? No, lo hago... por mí. Porque... sí, tengo que... pues tengo el hijo que me encanta y tengo el trabajo que me encanta. Las dos cosas, y las quiero compaginar.» (GD10_064_lingüista_teletrabajo tiempo completo)

Estas citas muestran un continuo esfuerzo contra los horarios y las demandas impuestas por las compañías y los/as empleadores/as. Las prácticas cotidianas vinculadas con el teletrabajo pueden ofrecer un nuevo contenido para la relación entre lo laboral y lo doméstico. Hemos incluido la noción de «resistencia» en el epígrafe de este apartado porque integra todo el conjunto descrito en el análisis de reflexiones, acciones y dinámicas de rechazo, oposición y crítica a modelos impuestos que las perjudican y de prácticas y acciones propositivas que gravitan sobre la oportunidad de la autoorganización y sobre la base de los intereses y necesidades de la trabajadora. Con esta dinámica aparece la idea de libertad, como hemos mencionado, pero también la autonomía, la responsabilidad y, no en menor medida, el compromiso, con los objetivos de la organización empleadora y con sus necesidades. Todo esto, aparte de beneficiar la práctica laboral y personal de la teletrabajadora, revierte, según ellas mismas afirman, en un aumento de su productividad y en una mayor implicación y compromiso con su organización.

«... A veces es mejor, claro, lo que decimos de trabajar por objetivos, trabajar por proyectos o por cosas, a veces lo puedes manejar más... mejor, $\mathrm{mmm}$... con un teletrabajo, ¿no? Y... y que también debería ser una de las razones más del teletrabajo, no solo la conciliación, sino el poder ser más eficaz, con más tiempo... con menos tiempo...» (GD2_072_periodista_teletrabajo parcial) 


\section{Conclusiones}

Como hemos mostrado en el anterior apartado, el teletrabajo debe ser comprendido como algo más que una técnica para mejorar nuestras maneras de organizar lo laboral o para mejorar la conciliación. Es un tipo de lógica o dispositivo que resignifica las prácticas que despliegan los/as teletrabajadores y que conducen a la creación de nuevos significados de lo profesional y de la compatibilización con la vida doméstica, familiar y personal. Así, los discursos de nuestras entrevistadas muestran cómo juegan un papel activo en el uso y la apropiación del teletrabajo. Del mismo modo, son capaces de redefinir la frontera entre la esfera laboral y la familiar y doméstica a partir de modelos de articulación propios, es decir, que emergen de sus prácticas organizativas más cotidianas y, también, elaborar un discurso crítico con el modelo laboral imperante. Su rechazo al modelo presencial, tan típico en nuestro país, con sus largas jornadas laborales, es total; y aunque no lo hemos mencionado, lo conectan con un marco de pensamiento y acción completamente patriarcal que privilegia al hombre en la esfera pública y tiende a relegar a la mujer a la doméstica. En ese sentido, ellas, de un modo consciente y crítico, vindican su papel como profesionales y como madres, sin querer renunciar a ninguno de ellos y hacen uso del teletrabajo como herramienta para denunciar un mercado laboral que excluye todo lo relacionado con la esfera del cuidado.

\section{Bibliografía}

ALINA-MIHAELA, D.; TUCLEA, C.E.; VRÂNCEANU, D.M.; TIGU, G. (2019). «Sustainable Social and Individual Implications of Telework: A New Insight into the Romanian Labor Market». Sustainability. Vol.11, núm. 13, pág. 1-12. https://doi.org/10.3390/su11133506.

BATTISTI, M.; VALLANTI, G. (2013). «Flexible wage contracts, temporary jobs, and firm performance: Evidence from Italian firms». Industrial Relations. Vol. 52, núm. 3, pág. 737-764. https://doi.org/10.1111/irel.12031.

EUROFOUND (2017). Worklife Balance and Flexible Working Arrangements in the European Union. Dublin: Eurofound.

EUROSTAT (2016). Eurostat. Your Key to European statistics. European Commission. Disponible en: https:// ec.europa.eu/eurostat/statistics-explained/index.php/Quality_of_life_indicators_-_productive_or_main_activity

FREENEY, M.K.; STRITCH, J.M. (2017). "Family-friendly policies, gender, and work-life balance in public sector». Review of Public Personnel Administration. Vol. 39, núm. 3, pág. 422-448. https://doi. org/10.1177/0734371X17733789.

GÁLVEZ, A.; TIRADO, F.; ALCÁRAZ, J. (2018). «Resisting long working hours: the case of spanish female teleworkers. German Journal of Human Resource Management. Vol. 32, núm. 3-4, pág. 195-216. https://doi. org/10.1177/2397002218782174.

GÁLVEZ, A.; TIRADO, F.; ALCÁRAZ, J. (2019). "Oh! Teleworking!; Regimes of engagement and the lived experience of (female) Spanish teleworkers». Business Ethics: A European Review. Vol. 29, pág. 180-192. https:// doi.org/10.1111/beer.12240.

HILBRECHT, M.; SHAW, S.; JOHNSON, L.; ANDREY, J. (2013). «Remixing work, family and leisure: Teleworkers' experiences of everyday life». New Technology, Work \& Employment. Vol. 28, núm. 2, pág. 130-144. https:// doi.org/10.1111/ntwe.12010.

KATTENBACH, R.; DEMEROUTI, E.; NACHREINER, F. (2010). «Flexible working times: Effects on employees' exhaustion, work-nonwork conflict and job performance». Career Development International. Vol. 15, núm. 3, pág. 279-295. https://doi.org/10.1108/13620431011053749.

KOSSEK, E.; LAUTSCH, B. (2017). «Work-Life Flexibility for Whom? Occupational Status and Work-Life Inequality in Upper, Middle, and Lower Level Jobs». Academy of Management Annals. Vol. 12, núm. 1, pág. 1-76. https://doi.org/10.5465/annals.2016.0059. 
KOTTEY, B.; SHARMA, B. (2016). "Predictors of flexible working arrangement provision in small and medium enterprises (SMEs) ». The International Journal of Human Resource Management. Vol. 27, núm. 22, pág. 2753-2770. https://doi.org/10.1080/09585192.2015.1102160.

LEWIS, S.; BEAUREGARD, T.A. (2018). «The meanings of work-life balance: a cultural perspective». En: JOHNSON, R.; SHEN, W.; SHOCKLEY, K.M. (eds). The Cambridge Handbook of the Global Workfamily Interface, Cambridge University Press, Cambridge, pág. 720-732. https://doi.org/10.1017/9781108235556.039.

LISTER, K.; HARNISH, T. (2010). Workshifting benefits: The bottom line. Telework Research Network/ Aerodite, Inc. http://www.workshifting.com/downloads/downloads/ Workshifting\%20Benefits-The\%20Bottom\%20 Line.pdf (consultado el 1 de septiembre de 2014).

MALENFANT, R. (2009). «Risk, Control and Gender: Reconciling Production and Reproduction in the Risk Society». Organization Studies. Vol. 30, núm. 2-3, pág. 205-226. https://doi.org/10.1177/0170840608101477.

SORENSEN, C. (2011). Enterprise Mobility: Tiny Technology with Global Impact on Work. Basingstoke: Palgrave Macmillan.

SORENSEN, C. (2017). "The performativity of choice: postfeminist perspective on work-life balance». Gender, Work and Organisation. Vol. 24, núm. 3, pág. 297-313. https://doi.org/10.1111/gwao.12163.

TIETZE, S.; MUSSON, G. (2010). Identity, identity work and the experience of working from home. Journal of Management Development. Vol. 29, núm. 2, pág. 148-156. https://doi.org/10.1108/02621711011019288.

WHEATLEY, D. (2012). «Good to Be Home? Time-Use and Satisfaction Levels among Home-Based Teleworkers». New Technology, Work and Employment. Vol. 27, núm. 3, pág. 224-241. https://doi.org/10.1111/j.1468005X.2012.00289.X.

WORLD AT WORK (2015). Trends in workplace flexibility. Scottsdale, AZ: World at Work Customer Relations.

YAO, J.; TAN, N.; ILIES, R. (2017). Telecommuting and work-family conflict: The Moderating role of work-family integration. Academy of Management Proceedings. Vol. 27, núm. 1, pág. 1-1. https://doi.org/10.5465/ AMBPP.2017.13717abstract.

Cita recomendada: GÁLVEZ, Ana. Una modalidad en auge. Mujeres y teletrabajo: más allá de la conciliación de la vida laboral y personal. Oikonomics [en línea]. Mayo 2020, n. 13, pp. 1-9. ISSN: 2339-9546. DOI: https://doi.org/10.7238/o.n13.2006 


\section{Ana Gálvez Mozo}

agalvez@uoc.edu

Profesora agregada de los Estudios de Psicología y Ciencias de la educación.

Ana Gálvez es Doctora en Psicología. Trabaja como profesora Agregada de Psicología Social del Trabajo y las Organizaciones en la titulación de Psicología. Fue directora del programa de Psicología desde el año 2011 hasta el 2017. Ha sido profesora de Psicología Social en la Universitat Autónoma de Barcelona y ha trabajado en diferentes organizaciones del sector profesional en ámbitos relacionados con la promoción de empleo y el diseño de formación en empresas a través de las TIC. Coordina el grupo de investigación TRAGSO (Trabajo, Género y Sociedad), cuyas líneas de investigación se centran en analizar la relación que se establece entre Género, Trabajo y Conciliación de la vida laboral, familiar y personal, y en explorar cómo los nuevos modelos laborales, caracterizados por la precariedad y la flexibilidad laboral, afectan y configuran la vida de los y las trabajadores/as y sus proyectos de vida. Ha participado y dirigido diferentes proyectos de investigación competitivos sobre estas temáticas.

Los textos publicados en esta revista están sujetos -si no se indica lo contrario- a una licencia de Reconocimiento 4.0 Internacional de Creative Commons. Puede copiarlos, distribuirlos, comunicarlos públicamente, hacer obras derivadas siempre que reconozca los créditos de las obras (autoría, nombre de la revista, institución editora) de la manera especificada por los autores o por la revista. La licencia completa se puede consultar en https://creativecommons.org/licenses/by/4.0/deed.es_ES.

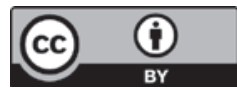

